\title{
DNA methylation signature of human hippocampus in Alzheimer's disease is linked to neurogenesis
}

\author{
Miren Altuna ${ }^{1,2}$, Amaya Urdánoz-Casado1, Javier Sánchez-Ruiz de Gordoa 1,2, María V. Zelaya ${ }^{3}$, Alberto Labarga ${ }^{4}$, \\ Julie M. J. Lepesant ${ }^{5}$, Miren Roldán ${ }^{1}$, Idoia Blanco-Luquin ${ }^{1}$, Álvaro Perdones ${ }^{4}$, Rosa Larumbe ${ }^{1,2}$, Ivonne Jericó ${ }^{2}$, \\ Carmen Echavarri ${ }^{1,2}$, Iván Méndez-López ${ }^{1,6}$, Luisa Di Stefano ${ }^{5}$ and Maite Mendioroz ${ }^{1,2^{*}}$ (D)
}

\begin{abstract}
Background: Drawing the epigenome landscape of Alzheimer's disease (AD) still remains a challenge. To characterize the epigenetic molecular basis of the human hippocampus in $A D$, we profiled genome-wide DNA methylation levels in hippocampal samples from a cohort of pure AD patients and controls by using the Illumina 450K methylation arrays.

Results: Up to 118 AD-related differentially methylated positions (DMPs) were identified in the AD hippocampus, and extended mapping of specific regions was obtained by bisulfite cloning sequencing. AD-related DMPs were significantly correlated with phosphorylated tau burden. Functional analysis highlighted that AD-related DMPs were enriched in poised promoters that were not generally maintained in committed neural progenitor cells, as shown by ChiP-qPCR experiments. Interestingly, AD-related DMPs preferentially involved neurodevelopmental and neurogenesisrelated genes. Finally, InterPro ontology analysis revealed enrichment in homeobox-containing transcription factors in the set of AD-related DMPs.
\end{abstract}

Conclusions: These results suggest that altered DNA methylation in the AD hippocampus occurs at specific regulatory regions crucial for neural differentiation supporting the notion that adult hippocampal neurogenesis may play a role in AD through epigenetic mechanisms.

Keywords: DNA methylation, Alzheimer's, Hippocampus, Adult neurogenesis, Poised promoters, Homeobox, Neurodevelopment, Epigenetics

\section{Background}

Alzheimer's disease (AD) is the leading cause of age-related dementia and one of the major global challenges of our time [1]. As knowledge about AD increases, so does the appreciation of the pathogenic complexity of the disorder [2]. Currently, $\mathrm{AD}$ is considered a complex disease that arises from the interaction between environmental and genetic factors [3], modulated through epigenetic mechanisms. Since epigenetics acts as an interface between the environment and the genome, a major focus has been upon

\footnotetext{
* Correspondence: maitemendilab@gmail.com; tmendioi@navarra.es 'Neuroepigenetics Laboratory, Navarrabiomed, Public University of Navarre (UPNA), IdiSNA (Navarra Institute for Health Research), c/ Irunlarrea, 3, 31008 Pamplona, Spain

2Department of Neurology, Complejo Hospitalario de Navarra, IdiSNA

(Navarra Institute for Health Research), Pamplona, Spain

Full list of author information is available at the end of the article
}

studying epigenetic alterations in $\mathrm{AD}$ to shed some light on the pathogenesis of the disease. DNA methylation is a major epigenetic modification that involves the attachment of a methyl group to the 5-carbon position of a cytosine residue and usually occurs at cytosine-guanine dinucleotides $(\mathrm{CpG})$. These $\mathrm{CpG}$ dinucleotides are clustered in the genome constituting $\mathrm{CpG}$ islands, which are enriched in the promoter of more than half of human genes and other important regulatory regions.

DNA methylation is known to be altered in complex diseases including $\mathrm{AD}$. Indeed, a number of gene-specific differences in DNA methylation have been reported so far [4-8]. More recently, genome-wide approaches have uncovered additional gene-specific methylation differences across different brain regions in AD $[9,10]$. By using Illumina Infinium HumanMethylation450K arrays, several

(C) The Author(s). 2019 Open Access This article is distributed under the terms of the Creative Commons Attribution 4.0 International License (http://creativecommons.org/licenses/by/4.0/), which permits unrestricted use, distribution, and 
genes have been found to be differentially methylated in AD brain autopsy samples, including some genes previously identified as harboring genetic variants for $A D$, such as ANK1 (ankyrin-1) or BIN1 (amphiphysin II) [11-14]. Importantly, a number of these DNA methylation marks are present in early stages of $\mathrm{AD}$, suggesting that such changes might play a role in the onset of the disorder [12]. On the whole, these reports are providing significant data to enrich our understanding of AD pathogenesis $[15,16]$.

So far, genome-wide DNA methylation studies on AD have been performed across different brain regions including prefrontal, frontal, and superior temporal neocortex, along with entorhinal cortex. In this study, we have taken a complementary strategy to profile genome-wide DNA methylation in the human hippocampus, a brain region particularly vulnerable to $\mathrm{AD}$ [17-19] and the core of pathological protein tau deposits [20]. We have applied Infinium HumanMethylation450 BeadChip array to hippocampal samples obtained from a homogeneous cohort of pure AD brains and controls. As a result, we report on novel gene-specific DNA methylation changes, recurrent across multiple affected subjects, which occur in the AD hippocampus. This DNA methylation signature of the $\mathrm{AD}$ hippocampus correlates with tau burden and also with specific changes in histone marks. Finally, in silico functional analysis of these changes points to molecular and biological alterations that may be especially relevant to the pathogenesis of $\mathrm{AD}$, including adult brain neurogenesis.

\section{Results}

\section{Hippocampal samples from AD patients and controls}

DNA methylation changes were evaluated in 36 postmortem hippocampal samples obtained from 26 patients with $\mathrm{AD}$ and 12 control subjects. To avoid spurious molecular findings related to multiprotein deposits, only $\mathrm{AD}$ cases with pure deposits of p-tau and $\beta$-amyloid were eligible for the study and controls were free of any protein aggregates. This approach maximizes the chances of finding true molecular associations with $\mathrm{AD}$, even though reducing the number of older controls.

Neuropathological and demographic features of subjects, including age, gender, ABC score, and postmortem interval (PMI), are listed in Additional file 1: Table S1. AD subjects were older than controls $(81.2 \pm 12.1$ versus $50.7 \pm 21.5 ; \quad p$ value $<0.01)$, and no differences were found regarding gender ( $p$ value $=0.16$ ). The PMI ranged from 1.4 to $33 \mathrm{~h}$ and were not significantly different between groups $(8.2 \pm 4.2 \mathrm{~h}$ in controls versus $7.9 \pm 7.1 \mathrm{~h}$ in AD samples; $p$ value $=0.91$.

Since DNA methylation may be affected by cellular composition of the brain samples, cell proportions were estimated by using the CETS $\mathrm{R}$ package as previously described $[14,21]$. No statistically significant differences were found in cell proportions between the AD group and the control group (control mean neuronal cell proportion $=0.18$ versus $\mathrm{AD}$ mean neuronal cell proportion $=0.20, p$ value $=0.64)$.

\section{Characterization of differentially methylated positions in the AD hippocampus}

Differential methylation analysis between the $A D$ and control hippocampus was performed by using the limma package (R/Bioconductor). After adjusting for age and false discovery rate (FDR) correction, the analysis revealed 118 AD-related differentially methylated positions (DMPs) (absolute $\beta$-difference $\geq 0.1$ and adjusted $p$ value $\leq 0.05$ ) located next to 159 genes (Fig. 1, Table 1). Most of the DMPs, 102 (86.4\%), were hypermethylated in AD cases compared to controls (Fig. 2ac). Inspection of methylation patterns revealed that AD-related DMPs showed a mild-to-moderate effect size, as the average absolute $\beta$-difference was $0.12(\mathrm{SD}=$ 0.02) (Fig. 2b). These results are in line with previous AD methylome studies, which showed recurrent gains in DNA methylation of a mild-to-moderate effect size in other brain regions [9-14]. Indeed, up to $17(10.7 \%)$ differentially methylated genes in our study are found among the top-ranked genes in previous $\mathrm{AD}$ methylome studies performed on frontal, temporal, or entorhinal cortex [11, 12, 14] (Additional file 1: Table S2).

Genomic distribution of AD-related DMPs was next analyzed. We observed that DMPs were more likely to locate in CpG islands, showing a 1.5-fold enrichment ( $p$ value $<0.001)$ compared with random expectation based on all probes included in the analysis. In addition, significant enrichment was found at first exon (2.4-fold, $p$ value $<0.001)$ and body $(1.2$-fold, $p$ value $<0.05)$ regions (Fig. 2d). Moreover, up to $13(8.1 \%)$ genes were associated with 2 or more AD-related DMPs which would suggest the presence of hotspots of aberrant methylation gain in the human hippocampus affected by AD.

\section{Validation and additional mapping of DMPs}

Among the 118 AD-related DMPs, we selected 7 DMPs to be validated based on their genomic location in or near genes relevant to brain function or AD pathology, including some of the candidate hotspots, i.e., $H A N D 2$, HOXA3, HIST1H3E, NXN, PAX3, RBMS1, and RHOB. All the selected genes were successfully validated by bisulfite cloning sequencing since a significant correlation was shown between the $450 \mathrm{~K}$ array data and methylation levels obtained by bisulfite cloning sequencing (Additional file 1: Table S3). Additional mapping of the altered methylation pattern across multiple contiguous CpGs was generated (Fig. 2g and Fig. 3, Additional file 1: 


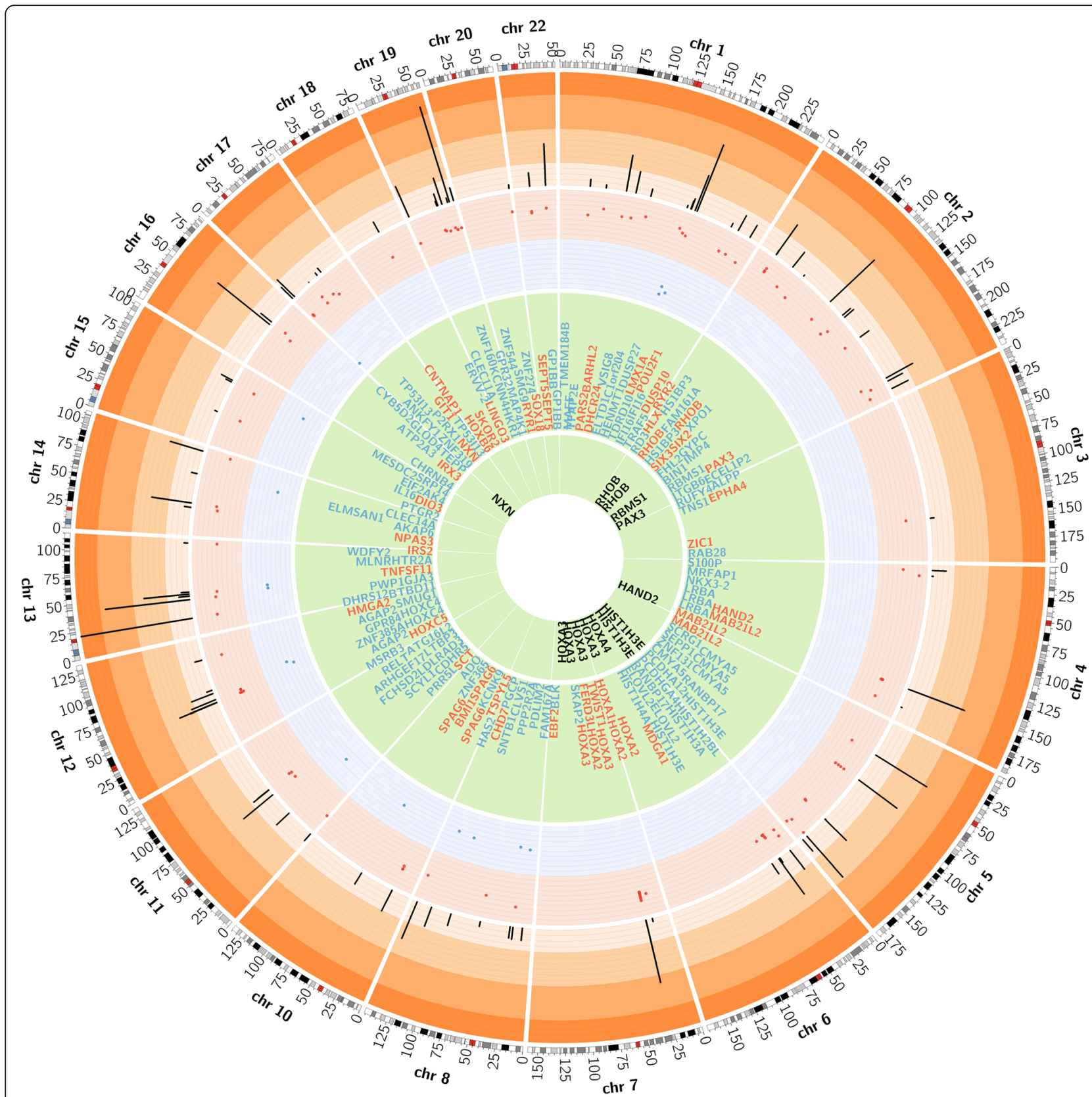

Fig. 1 CIRCOS plot of differentially methylated positions (DMPs) in AD hippocampus. The CIRCOS plot shows a summary of DNA methylation screening results in the $\mathrm{AD}$ hippocampus and its validation by bisulfite cloning sequencing. The perimeter of the circular figure represents the human chromosomes, showing the cytogenetic bands and centromeres (in red). Only those chromosomes harboring DMPs are represented in the painted circles. $X$ and $Y$ chromosomes were excluded from the analysis. The orange circle represents $p$ value for each DMP. The inner red and blue dots represent the results of the differential analysis (beta difference) for each DMPs, including gains in methylation (red dots) and losses in methylation (blue dots). The next green circle reports the names of the genes associated to each DMPs. Those genes associated with neurogenesis are highlighted in red font. In black font, those genes that were validated by bisulfite cloning sequencing are shown

Figure S1). The results of this mapping support the idea that changes in DNA methylation are not confined to the CpGs queried by the array. On the contrary, alterations of DNA methylation in AD seem to be consistently distributed across discrete regions of the genome and to involve multiple contiguous CpGs.

\section{DNA methylation levels correlate with phosphorylated tau protein burden}

The human hippocampus is particularly vulnerable to specific anatomopathological changes in $\mathrm{AD}$ and is considered the region where tau pathology initiates, together with the entorhinal cortex [17-19]. To explore whether 
Table 1 Differentially methylated positions (DMPs) in AD hippocampus measured by 450K Illumina BeadChip array

\begin{tabular}{|c|c|c|c|c|c|c|c|c|c|}
\hline \multirow{2}{*}{$\frac{\text { DMPs }}{\text { cg02091185 }}$} & \multicolumn{2}{|c|}{ Genomic coordinates } & \multirow{2}{*}{$\begin{array}{l}\text { Beta. difference } \\
0.198\end{array}$} & \multirow{2}{*}{$\frac{\text { FDR } p \text { value }}{0.003}$} & \multirow{2}{*}{$\begin{array}{l}\text { GenelD1 } \\
\text { RANBP17 }\end{array}$} & \multirow[t]{2}{*}{ GenelD2 } & \multirow{2}{*}{$\begin{array}{l}\text { Neurogenesis-related } \\
\text { No }\end{array}$} & \multicolumn{2}{|c|}{ Neurogenesis-related PMID } \\
\hline & 5 & 170288766 & & & & & & & \\
\hline cg07333191 & 4 & 13526769 & 0.168 & 0.002 & RAB28 & NKX3-2 & No & & \\
\hline cg14780466 & 2 & 20870812 & 0.150 & 0.011 & GDF7 & APOB & Yes & 17293457 & 22897442 \\
\hline cg04370442 & 16 & 58019866 & 0.147 & 0.013 & TEPP & ZNF319 & No & & \\
\hline cg16867657 & 6 & 11044877 & 0.145 & 0.002 & ELOVL2 & & No & & \\
\hline cg04154027 & 5 & 78985588 & 0.144 & 0.002 & CMYA5 & & No & & \\
\hline cg19506623 & 2 & 161265259 & 0.143 & 0.036 & RBMS1 & ITGB6 & No & & \\
\hline cg04498198 & 17 & 27899966 & 0.140 & 0.002 & TP53113 & GIT1 & Yes & & 25792865 \\
\hline cg23077820 & 2 & 223154176 & 0.140 & 0.002 & PAX3 & EPHA4 & Yes & 26287727 & 25978062 \\
\hline cg11015251 & 7 & 27170554 & 0.138 & 0.005 & HOXA4 & & No & & \\
\hline cg16258854 & 2 & 20648194 & 0.138 & 0.005 & $\mathrm{RHOB}$ & HS1BP3 & Yes & 15306568 & \\
\hline cg01463828 & 8 & 22446721 & 0.137 & 0.007 & PDLIM2 & & No & & \\
\hline cg13935577 & 12 & 107974897 & 0.136 & 0.008 & BTBD11 & PWP1 & No & & \\
\hline cg12100751 & 1 & 109203672 & 0.134 & 0.002 & HENMT1 & & No & & \\
\hline cg19022697 & 1 & 55247140 & 0.133 & 0.004 & PARS2 & DHCR24 & Yes & 29410512 & 24842139 \\
\hline cg25840926 & 2 & 20647987 & 0.133 & 0.002 & $\mathrm{RHOB}$ & HS1BP3 & Yes & 15306568 & \\
\hline cg22962123 & 7 & 27153605 & 0.131 & 0.008 & HOXA3 & HOXA2 & Yes & 12954718 & 10230789 \\
\hline cg05637536 & 1 & 154475068 & 0.130 & 0.002 & TDRD10 & & No & & \\
\hline cg01331772 & 2 & 131094827 & 0.129 & 0.002 & IMP4 & & No & & \\
\hline cg23279355 & 5 & 78985592 & 0.128 & 0.002 & CMYA5 & & No & & \\
\hline cg01579024 & 5 & 170288757 & 0.127 & 0.013 & RANBP17 & & No & & \\
\hline cg15548613 & 22 & 38610795 & 0.127 & 0.002 & MAFF & TMEM184B & No & & \\
\hline cg13172549 & 7 & 27153636 & 0.125 & 0.019 & HOXA3 & HOXA2 & Yes & 12954718 & 10230789 \\
\hline cg02231404 & 20 & 62679635 & 0.124 & 0.008 & SOX18 & & Yes & 29666335 & \\
\hline cg13327545 & 10 & 22623548 & 0.124 & 0.002 & SPAG6 & BMI1 & Yes & 29666335 & 19212323 \\
\hline cg18247055 & 10 & 22634226 & 0.122 & 0.003 & SPAG6 & & Yes & 26130477 & \\
\hline cg00921266 & 7 & 27153663 & 0.122 & 0.039 & HOXA3 & HOXA2 & Yes & 12954718 & 10230789 \\
\hline cg09490371 & 2 & 233253024 & 0.120 & 0.011 & ECEL1P2 & ALPP & No & & \\
\hline cg17448336 & 3 & 147141588 & 0.119 & 0.047 & $\mathrm{ZIC1}$ & & Yes & 17507568 & \\
\hline cg16127683 & 15 & 40268777 & 0.119 & 0.021 & EIF2AK4 & SRP14 & No & & \\
\hline cg25774643 & 11 & 627175 & 0.119 & 0.028 & $\mathrm{SCT}$ & CDHR5 & Yes & 21159798 & \\
\hline cg18121224 & 5 & 176559563 & 0.119 & 0.010 & NSD1 & & No & & \\
\hline cg07816556 & 6 & 26017280 & 0.119 & 0.004 & HIST1H4A & HIST1H3A & No & & \\
\hline cg16308533 & 17 & 40838983 & 0.118 & 0.004 & CNTNAP1 & EZH1 & Yes & 26740489 & 23932971 \\
\hline cg04533276 & 22 & 19709548 & 0.117 & 0.007 & SEPT5 & GP1BB & Yes & 17935997 & \\
\hline cg22900415 & 13 & 20736075 & 0.117 & 0.010 & GJA3 & & No & & \\
\hline cg22385702 & 2 & 45175881 & 0.116 & 0.025 & SIX3 & $\mathrm{SIX} 2$ & Yes & 17576749 & 11401394 \\
\hline cg09655403 & 5 & 78985495 & 0.116 & 0.004 & CMYA5 & & No & & \\
\hline cg22507154 & 1 & 91185233 & 0.116 & 0.016 & BARHL2 & & Yes & 22307612 & \\
\hline cg24369989 & 15 & 78933807 & 0.115 & 0.002 & CHRNB4 & & No & & \\
\hline cg16404157 & 14 & 38724648 & 0.115 & 0.010 & CLEC14A & & No & & \\
\hline cg03146625 & 12 & 54448729 & 0.115 & 0.018 & $\mathrm{HOXC4}$ & SMUG1 & No & & \\
\hline cg04027736 & 7 & 27143403 & 0.115 & 0.011 & HOXA2 & & Yes & 10230789 & \\
\hline cg12024906 & 19 & 37825679 & 0.115 & 0.005 & HKR1 & & No & & \\
\hline
\end{tabular}


Table 1 Differentially methylated positions (DMPs) in AD hippocampus measured by 450K Illumina BeadChip array (Continued)

\begin{tabular}{|c|c|c|c|c|c|c|c|c|c|}
\hline \multirow{2}{*}{$\frac{\text { DMPs }}{\text { cg00303378 }}$} & \multicolumn{2}{|c|}{ Genomic coordinates } & \multirow{2}{*}{$\begin{array}{l}\text { Beta. difference } \\
0.115\end{array}$} & \multirow{2}{*}{$\frac{\text { FDR } p \text { value }}{0.002}$} & \multirow{2}{*}{$\frac{\text { GenelD1 }}{\text { VSIG8 }}$} & \multirow{2}{*}{$\frac{\text { GenelD2 }}{\text { C1orf204 }}$} & \multirow{2}{*}{$\begin{array}{l}\text { Neurogenesis-related } \\
\text { No }\end{array}$} & \multicolumn{2}{|c|}{ Neurogenesis-related PMID } \\
\hline & 1 & 159825552 & & & & & & & \\
\hline cg17508941 & 7 & 19183280 & 0.114 & 0.002 & TWIST1 & FERD3L & Yes & 23555309 & 23254923 \\
\hline cg24079702 & 2 & 106015771 & 0.114 & 0.005 & FHL2 & & No & & \\
\hline cg15084543 & 1 & 79472408 & 0.113 & 0.006 & ELTD1 & & No & & \\
\hline cg25738176 & 17 & 3848506 & 0.113 & 0.033 & ATP2A3 & $\mathrm{P} 2 \mathrm{RX} 1$ & No & & \\
\hline cg20864214 & 11 & 73054121 & 0.113 & 0.018 & RELT & ARHGEF17 & No & & \\
\hline cg06452665 & 13 & 43148436 & 0.113 & 0.010 & TNFSF11 & & Yes & 24087792 & \\
\hline cg21572722 & 6 & 11044894 & 0.113 & 0.003 & ELOVL2 & & No & & \\
\hline cg26092675 & 6 & 26225258 & 0.112 & 0.002 & HIST1H3E & & No & & \\
\hline cg07336350 & 16 & 54322127 & 0.111 & 0.013 & $\mathrm{IRX} 3$ & & Yes & 10704856 & \\
\hline cg07809484 & 19 & 51231968 & 0.111 & 0.002 & GPR32 & CLEC11A & No & & \\
\hline cg16651126 & 7 & 27170552 & 0.111 & 0.007 & HOXA4 & & No & & \\
\hline cg05726109 & 22 & 19709755 & 0.111 & 0.007 & SEPT5 & GP1BB & Yes & 17935997 & \\
\hline cg07584855 & 1 & 221055545 & 0.110 & 0.007 & HLX & DUSP10 & Yes & 7907015 & 19139271 \\
\hline cg14566959 & 5 & 140772681 & 0.110 & 0.006 & PCDHGA4 & & No & & \\
\hline cg02798280 & 19 & 39087135 & 0.110 & 0.007 & MAP4K1 & RYR1 & No & & 17767953 \\
\hline cg24756378 & 14 & 33401638 & 0.110 & 0.027 & NPAS3 & AKAP6 & Yes & 21709683 & \\
\hline cg11864574 & 10 & 22635028 & 0.110 & 0.015 & SPAG6 & & Yes & 29666335 & \\
\hline cg06555959 & 8 & 61835620 & 0.110 & 0.050 & CLVS1 & CHD7 & Yes & & 23827709 \\
\hline cg12253175 & 12 & 58132093 & 0.110 & 0.029 & AGAP2 & & No & & \\
\hline cg15834355 & 12 & 54442075 & 0.110 & 0.014 & $\mathrm{HOXC4}$ & $\mathrm{HOXC5}$ & Yes & & 23103965 \\
\hline cg09596958 & 12 & 58132105 & 0.109 & 0.031 & AGAP2 & & No & & \\
\hline cg02267270 & 6 & 37616410 & 0.109 & 0.047 & MDGA1 & CCDC167 & Yes & 21104742 & \\
\hline cg18181229 & 1 & 164545699 & 0.109 & 0.016 & PBX1 & LMX1A & Yes & 27226325 & 24172139 \\
\hline cg01421119 & 1 & 211555733 & 0.109 & 0.019 & TRAF5 & RD3 & No & & \\
\hline cg03729251 & 4 & 151501035 & 0.108 & 0.012 & LRBA & MAB21L2 & Yes & & 11960703 \\
\hline cg22090150 & 17 & 4098227 & 0.107 & 0.002 & ANKFY1 & CYB5D2 & No & & \\
\hline cg05877788 & 17 & 27899874 & 0.107 & 0.003 & TP53113 & GIT1 & Yes & & 25792865 \\
\hline cg06396119 & 13 & 49792767 & 0.106 & 0.025 & MLNR & & No & & \\
\hline cg22154659 & 7 & 27134369 & 0.106 & 0.015 & HOXA1 & SKAP2 & Yes & 14522873 & \\
\hline cg14266527 & 4 & 151501298 & 0.106 & 0.004 & LRBA & MAB21L2 & Yes & & 11960703 \\
\hline cg24177393 & 5 & 43037517 & 0.105 & 0.033 & SEPP1 & ZNF131 & No & & \\
\hline cg07942135 & 7 & 27154262 & 0.105 & 0.022 & HOXA3 & HOXA2 & Yes & 12954718 & 10230789 \\
\hline cg22904711 & 19 & 44278628 & 0.105 & 0.041 & KCNN4 & SMG9 & No & & \\
\hline cg01566965 & 4 & 174447847 & 0.104 & 0.028 & HAND2 & SCRG1 & Yes & 22323723 & \\
\hline cg14557699 & 5 & 140254909 & 0.104 & 0.002 & PCDHA12 & & No & & \\
\hline cg26698460 & 19 & 58716004 & 0.104 & 0.010 & ZNF274 & ZNF544 & No & & \\
\hline cg02287710 & 14 & 102027660 & 0.103 & 0.004 & $\mathrm{DlO} 3$ & & Yes & 27707971 & \\
\hline cg21415530 & 8 & 140715802 & 0.103 & 0.010 & KCNK9 & & No & & \\
\hline cg07589899 & 2 & 62020677 & 0.103 & 0.002 & XPO1 & FAM161A & No & & \\
\hline cg20192747 & 18 & 44774846 & 0.103 & 0.003 & SKOR2 & & Yes & 24491816 & \\
\hline cg17179862 & 17 & 46681362 & 0.103 & 0.003 & HOXB6 & LOC404266 & Yes & 10686603 & \\
\hline cg26587870 & 6 & 27730563 & 0.103 & 0.010 & ZNF184 & HIST1H2BL & No & & \\
\hline cg01089914 & 2 & 218843229 & 0.102 & 0.017 & RUFY4 & TNS1 & No & & \\
\hline
\end{tabular}


Table 1 Differentially methylated positions (DMPs) in AD hippocampus measured by 450K Illumina BeadChip array (Continued)

\begin{tabular}{|c|c|c|c|c|c|c|c|c|}
\hline \multirow{2}{*}{$\frac{\text { DMPs }}{\text { cg14962509 }}$} & \multicolumn{2}{|c|}{ Genomic coordinates } & \multirow{2}{*}{$\begin{array}{l}\text { Beta. difference } \\
0.102\end{array}$} & \multirow{2}{*}{$\frac{\text { FDR } p \text { value }}{0.004}$} & \multirow{2}{*}{$\frac{\text { GenelD1 }}{\text { TFAP2E }}$} & \multirow[t]{2}{*}{ GenelD2 } & \multirow{2}{*}{$\begin{array}{l}\text { Neurogenesis-related } \\
\text { No }\end{array}$} & Neurogenesis-related PMID \\
\hline & 1 & 36039655 & & & & & & \\
\hline cg21869609 & 19 & 2291613 & 0.102 & 0.018 & LINGO3 & & Yes & 18297755 \\
\hline cg08865099 & 7 & 27281581 & 0.102 & 0.043 & EVX1 & & Yes & 10399918 \\
\hline cg13836098 & 6 & 26225268 & 0.102 & 0.002 & HIST1H3E & & No & \\
\hline cg00611789 & 5 & 78985432 & 0.102 & 0.011 & CMYA5 & & No & \\
\hline $\operatorname{cg} 13771313$ & 11 & 72533295 & 0.102 & 0.017 & ATG16L2 & FCHSD2 & No & \\
\hline cg21811021 & 4 & 6659346 & 0.101 & 0.015 & S100P & MRFAP1 & No & \\
\hline $\operatorname{cg} 11254700$ & 19 & 53561386 & 0.101 & 0.020 & ERW-2 & ZNF160 & No & \\
\hline $\operatorname{cg} 14557202$ & 12 & 54764371 & 0.101 & 0.028 & ZNF385A & GPR84 & No & \\
\hline cg03422911 & 1 & 237205295 & 0.101 & 0.004 & RYR2 & & Yes & 17767953 \\
\hline cg19153828 & 2 & 127782651 & 0.101 & 0.009 & BIN1 & GYPC & No & \\
\hline cg06867571 & 11 & 65306934 & 0.101 & 0.019 & LTBP3 & SCYL1 & No & \\
\hline cg09317554 & 4 & 151505084 & 0.101 & 0.014 & LRBA & MAB21L2 & Yes & 11960703 \\
\hline cg05404236 & 13 & 110437093 & 0.100 & 0.017 & IRS2 & & Yes & 28833887 \\
\hline cg027711117 & 8 & 11279352 & -0.100 & 0.022 & FAM167A & BLK & No & \\
\hline cg10373891 & 13 & 52338758 & -0.101 & 0.017 & DHRS12 & WDFY2 & No & \\
\hline cg24607755 & 11 & 36171375 & -0.102 & 0.010 & LDLRAD3 & PRR5L & No & \\
\hline cg20102280 & 13 & 47470793 & -0.102 & 0.004 & HTR2A & & No & \\
\hline cg21735068 & 8 & 97975467 & -0.104 & 0.015 & PGCP & TSPYL5 & Yes & 26911678 \\
\hline cg23907053 & 12 & 70215816 & -0.107 & 0.015 & RAB3IP & MYRFL & No & \\
\hline cg14701867 & 10 & 64193068 & -0.107 & 0.023 & ZNF365 & ADO & No & \\
\hline cg14830371 & 8 & 25991602 & -0.108 & 0.027 & PPP2R2A & EBF2 & Yes & 25762221 \\
\hline cg16668651 & 15 & 81316319 & -0.110 & 0.035 & IL16 & MESDC2 & No & \\
\hline cg06688910 & 8 & 122466955 & -0.110 & 0.027 & SNTB1 & HAS2 & No & \\
\hline cg07463059 & 1 & 158979810 & -0.119 & 0.010 & IFI16 & & No & \\
\hline cg05165025 & 14 & 74253312 & -0.119 & 0.046 & PTGR2 & ELMSAN1 & No & \\
\hline cg07677157 & 12 & 66050928 & -0.133 & 0.026 & HMGA2 & MSRB3 & Yes & 18640244 \\
\hline cg11236550 & 1 & 167090757 & -0.138 & 0.012 & DUSP27 & POU2F1 & No & 18241856 \\
\hline cg20597486 & 1 & 158979841 & -0.151 & 0.002 & IFI16 & & No & \\
\hline cg19987768 & 17 & 750306 & -0.162 & 0.043 & NXN & GLOD4 & Yes & 29037191 \\
\hline
\end{tabular}

The table shows 118 DMPs with $\beta$-difference $>0.100$, prioritized by beta difference (delta) criteria. FDR false discovery rate, Adj. adjusted, ID identification, PMID PubMed identification. Each probe (CpG site) was annotated by UCSC hg19 build. The last two columns show the PMID of papers supporting the involvement of the genes into neurogenesis or neural development

the altered DNA methylation pattern reflects AD pathological changes in the hippocampus, we used a semi-automated quantitative method described in detail elsewhere [7] to measure the extension of phosphorylated tau (p-tau) deposits in our set of hippocampal samples. Next, Pearson's coefficient was calculated to evaluate the correlation between DNA methylation levels at each of the identified 118 AD-related DMPs and the extension of p-tau deposits in the hippocampus. We found that DNA methylation levels in 43 (36.4\%) DMPs were significantly correlated with the burden of p-tau deposits (Additional file 1: Table S4). The strongest correlation was observed for differentially methylated CpGs located close to the SOX18, HKR1, PCDHA12, and ATG16L2 genes.

\section{AD-related DMPs overlap bivalent histone marks and poised promoters}

Independent epigenetic mechanisms may play together to coordinately fine-tune gene expression. Therefore, we next asked whether the set of 118 AD-related DMPs was predicted to associate with other epigenetic features such as histone modifications. To this end, we first performed a functional in silico analysis for enrichment in histone marks using ENCODE/Broad data for human brain hippocampus, normal human astrocytes (NH-A), 

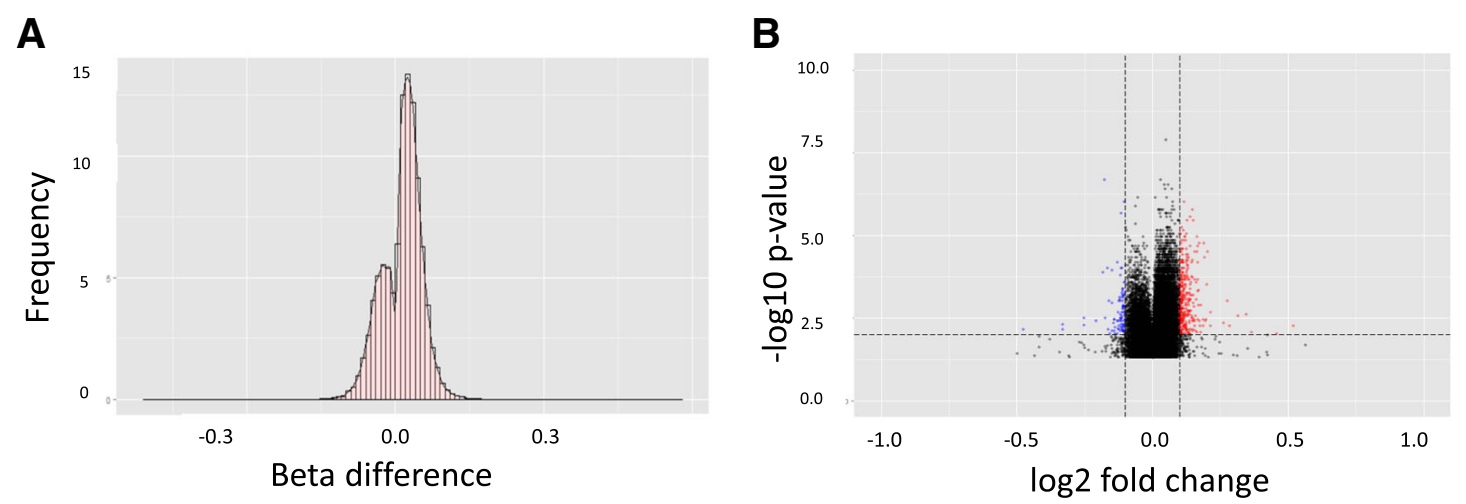

C

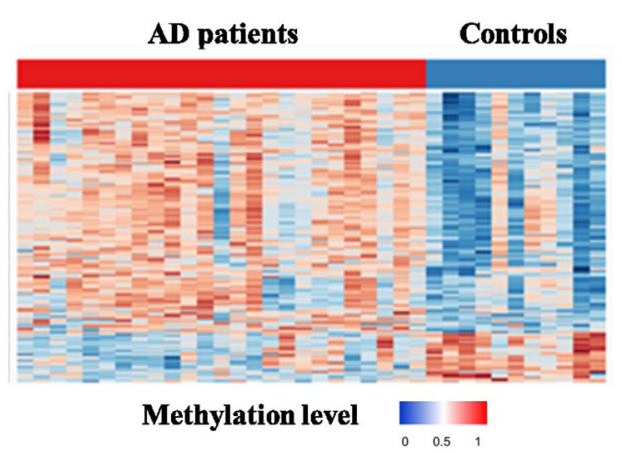

D

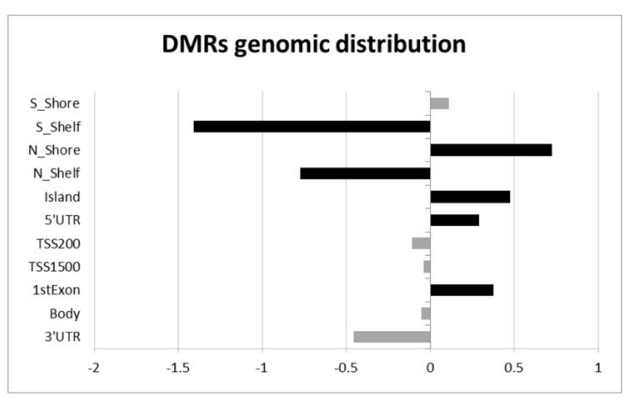

E

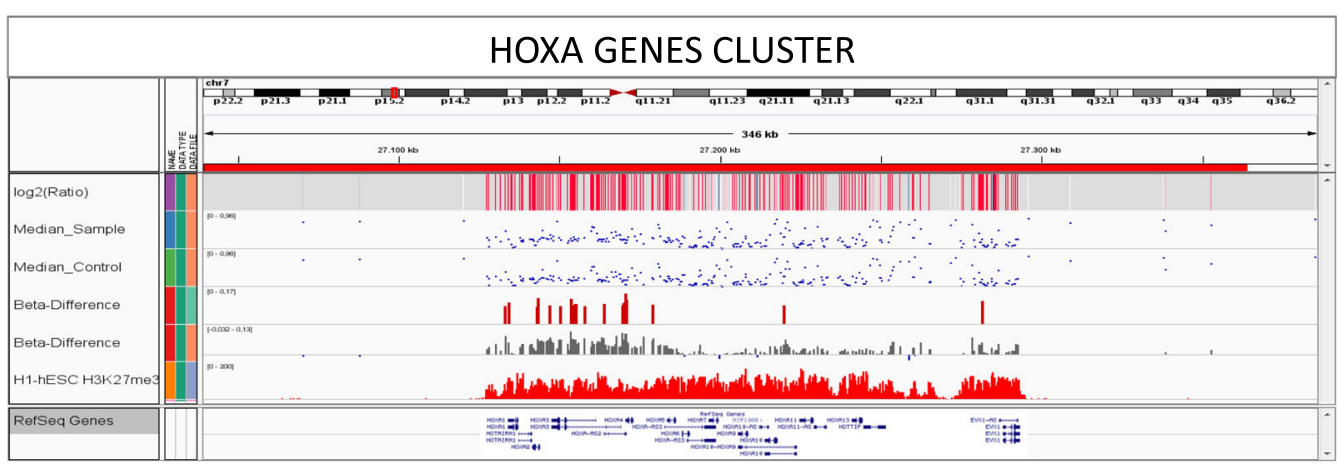

F
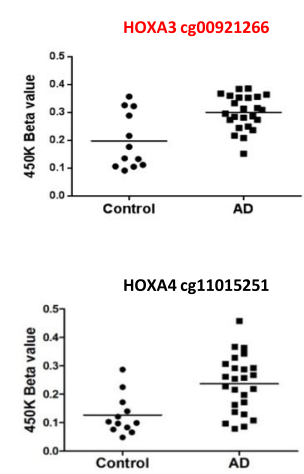
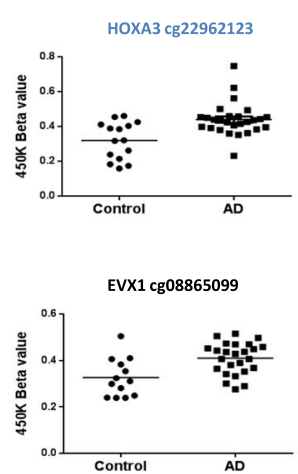

G
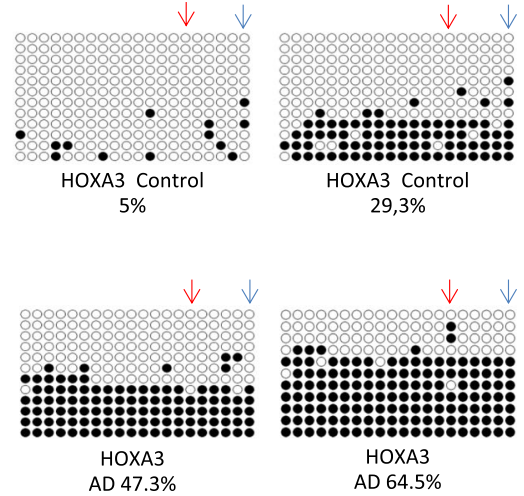

Fig. 2 (See legend on next page.) 


\begin{abstract}
(See figure on previous page.)
Fig. 2 Characterization of AD-related DMPs. a The histogram of beta difference value distribution per CpGs shows a clear bias toward the hypermethylated changes ( $>0.0)$ in AD hippocampal samples compared to controls. $\mathbf{b}$ The volcano plot shows a greater number of hypermethylated marks (red dots) compared to hypomethylated marks (blue dots) that crossed the statistical thresholds (dotted lines) in this study. The graph also shows that DNA methylation changes in AD hippocampus are mild to moderate in effect size. $\mathbf{c}$ The heat map graph reveals that most of the DNA methylation changes represent gains (red squares) in methylation. $\mathbf{d}$ Distribution of DMPs regarding gene structure. The bar graph shows the log2 ratios of observed (fraction of differentially methylated probes that overlap a particular region) to expected (fraction of probes selected for analysis that overlap a particular region). $\mathrm{S}=$ south, $\mathrm{N}=$ north, TSS = transcription start site. e Differential analysis revealed up to 8 AD-related DMPs located within the HOXA genes cluster in the short arm of chromosome 7. The upper tracks show 450K microarray values and results of differential analysis. Blue dots represent per CpG median $\beta$-values for patients and controls. Vertical red bars represent $\beta$-difference values for CpGs included in the DMPs that crossed the statistical threshold ( $\beta$ difference $>0.1$ and $p$ value $<0.05$ ). Grey bars represent $\beta$-difference values for the CpGs included in the DMPs. Methylation values are aligned to ENCODE/ Broad data for H3K27me3 histone marks in H1 human Embryonic Stem Cells (H1-hESC) at the bottom. $\mathbf{f}$ Dot plot graphs show 450K microarray $\beta$-values for the CpGs with most significant $p$ value for each 4 genes within DMPs in the HOXA cluster. The selected CpGs are as follow, HOXA2: cg04027736, HOXA3: cg22962123, HOXA4:cg16651126, EVX1:cg08865099. g Extended mapping of hypermethylated DMPs within HOXA3 gene in 2 AD cases (below) compared to 2 controls (above) obtained by bisulfite cloning sequencing that shows how differential methylation affects multiple contiguous CpGs. The amplicon overlaps cg00921266 (blue arrow) and cg22962123 (red arrow). Black circles represent methylated cytosines while white circles denote unmethylated cytosines. Each column symbolizes a unique CpG site in the examined amplicon, and each line represents an individual DNA clone. Average percentage of methylation for each analyzed sample (control or patient) at this particular amplicon is indicated at the bottom of each sample
\end{abstract}

and H1 human Embryonic Stem Cells (H1hESC) identified through the WashU Epigenome Browser [22] and the UCSC Genome Browser [23].

We found that AD-related DMPs were overrepresented in regions of repressive histone marks, particularly in H3K27me3 mark in H1hESCs and H3K9me3 in the human hippocampus (Fig. 4a). We also realized that many of the AD-related DMPs shared both repressive (H3K27me3) and activating (H3K4me2 and H3K4me3) marks in the same locus. In other words, these DMPs overlapped regions of bivalent chromatin that usually characterizes poised promoters. Genes with poised promoters are generally repressed but ready for immediate activation in response to certain signals [24], and are thought to be key developmentally regulated genes not only in stem cells [24-27] but also in differentiated cells [27-29]. In our study, 70 (59.3\%) AD-related DMPs overlapped poised promoters. When taking into account the direction of the methylation change, we observed that up to $68(66.6 \%)$ of the hypermethylated DMPs overlapped bivalent promoters, whereas only 2 (12.5\%) of the hypomethylated DMPs overlapped bivalent promoters (Fig. 4b).

\section{ChIP analysis of bivalent promoters in neural human progenitor cells}

Although most of the bivalent modifications in hESC are usually resolved during lineage commitment, a small subset of poised promoters in hESC may remain bivalent during differentiation [24, 27]. Indeed, bivalent promoters may play complex roles in differentiated cells, keeping some genes poised for activation $[24,29,30]$. To explore the fate of bivalent chromatin modifications in our set of AD-related DMPs, we performed a number of chromatin immunoprecipitation-quantitative PCR (ChIP-qPCR) experiments in neural human progenitor cells (NHPCs) which represent a more differentiated state than hESC in the brain tissue. We ran ChIP-qPCR assays on NHPCs using anti-H3K27me3 and antiH3K4me3 antibodies for selected differentially methylated genes, including the HOXA gene cluster. ChIP-qPCR analysis revealed that genes within the HOXA locus resolved to the repressive H3K27me3 status in NHPCs, as was also the case of the DIO3 and TDRD10 genes. On the contrary, ROHB, ELOVL2, and FHL2 resolved to H3K4me3 active status in the committed NHPCs. Only SEPT5 lost both H3K4me3 and H3K27me3 chromatin marks (Fig. 4c). These results illustrate a wide variety of histone marks fates for these differentially methylated genes which are poised in H1hESCs. Although additional research on this issue would be needed, these results suggest that AD-related DMPs are not enriched in genes that retain bivalent marks in lineage-committed cells.

\section{AD-related differentially methylated genes are linked to neural development and neurogenesis}

Next, we wanted to know whether the set of AD-related DMPs was enriched for genes involved in specific diseases, functions, and pathways. To this end, we performed different levels of analysis. First, manually curated search using PubMed revealed that 50 (42.4\%) AD-related DMPs were associated with genes related with neural development and neurogenesis (Table 1, Fig. 1). Consistently, most of these genes overlapped poised promoters (90.9\%), since they are key developmentally regulated genes.

Next, we used the Genomic Regions Enrichment of Annotations Tool (GREAT) [31] to identify enriched ontological terms in our set of AD-related DMPs. The enriched gene ontology (GO) terms in the biological process category revealed a set of different processes consistently associated with embryonic and brain morphogenesis among others (Additional file 1: Figure S2). In addition, our set of AD-related DMPs was enriched in 


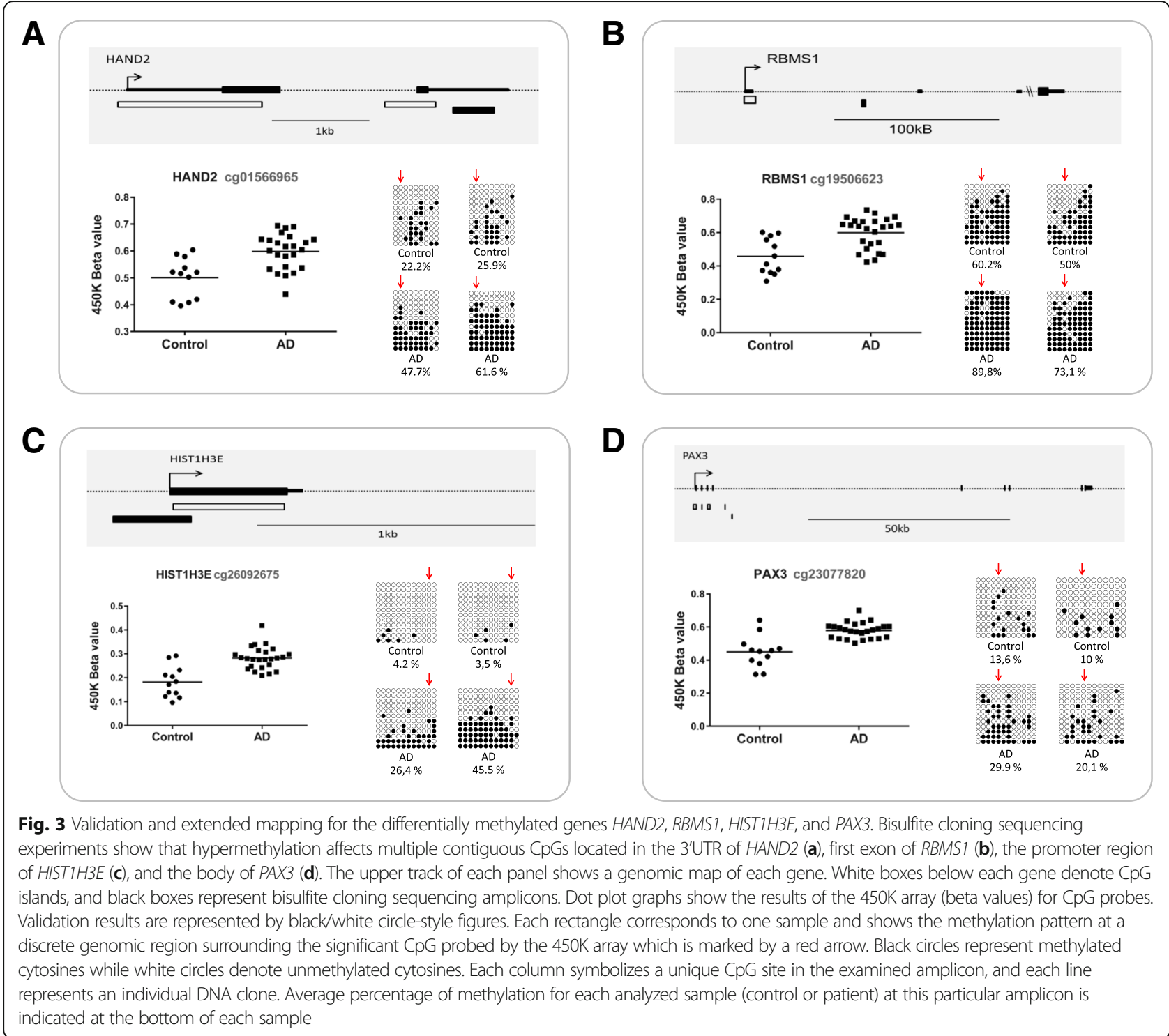

the "high mobility group (HMG) box domain binding" term in the GO molecular category (fold enrichment = 12.3; FDR $Q$-value <0.01). HMG is a protein domain which confers proteins the ability to bind DNA and is related to a number of DNA processes, including transcription and DNA repair [32].

Next, an InterPro ontology analysis was performed to test for protein domains, families, and functional sites. The analysis showed enrichment for homeobox domain-related terms in the set of AD-related DMPs (Additional file 1: Table S5). Homeobox transcription factors are crucial in regulating pluripotency and cellular differentiation [33].

\section{Discussion}

In this study, we profiled genome-wide DNA methylation levels to identify novel AD-related methylation changes in the human hippocampus. These results revealed genomic loci hypermethylated in $\mathrm{AD}$ cases compared to controls that largely overlap regulatory regions, mainly bivalent promoters. In addition, the DNA methylation signature was consistently related to genes crucial for neural development or neurogenesis and homeobox-containing transcription factors.

Neuropathological hallmarks of AD tend to occur in particularly vulnerable regions in the human brain $[17,20]$ and are associated to neuronal death and synapse loss from the very early stages of the disease [34]. However, molecular mechanisms underlying the specific brain region pattern of neuropathological changes in $\mathrm{AD}$ are not entirely clear. That was the rationale for selecting the human hippocampus to perform this epigenetic screening since it is a highly vulnerable region to $\mathrm{AD}$ neuropathological 


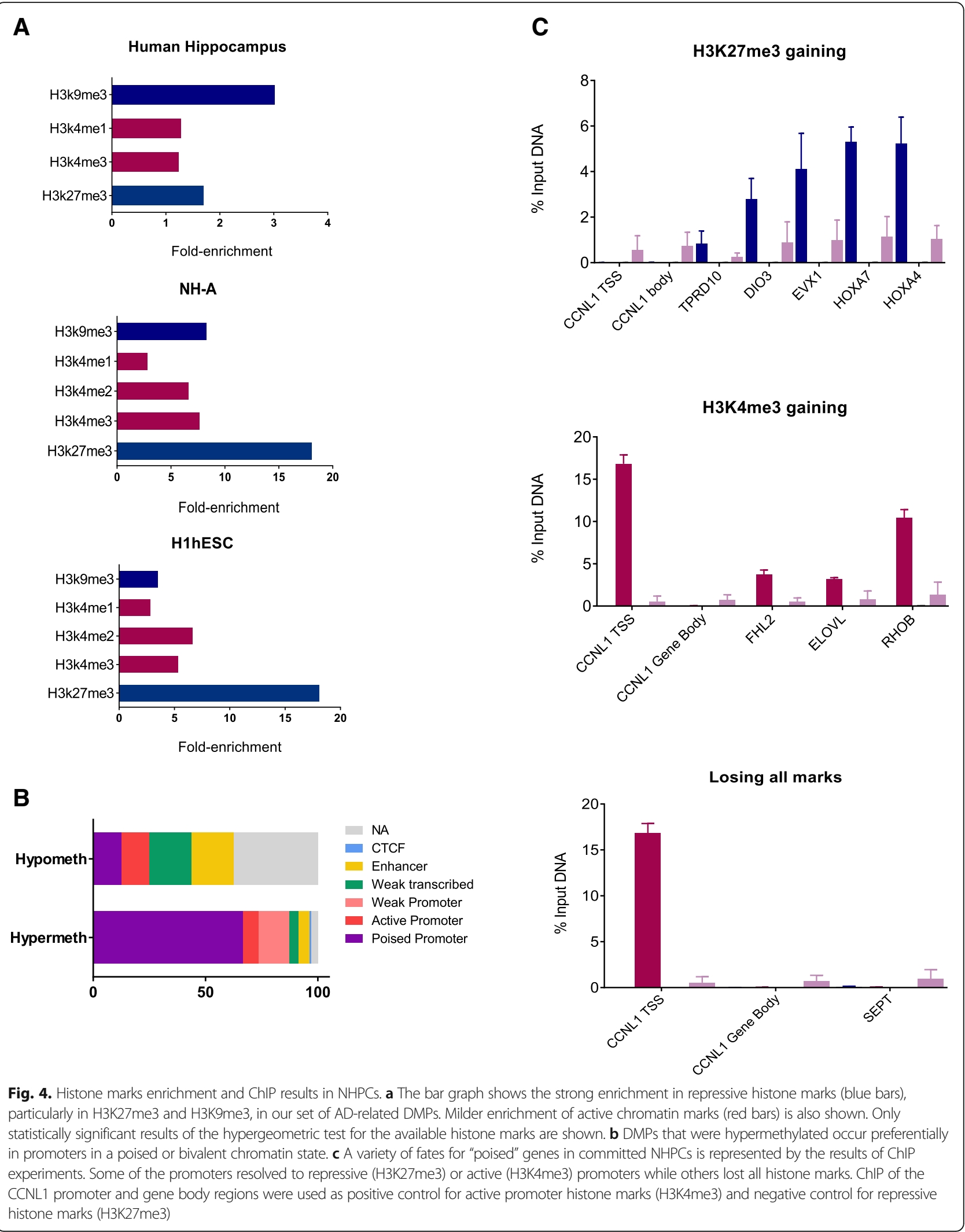


changes. In this regard, our analysis showed a statistically significant positive correlation between DNA methylation levels at $36.4 \%$ of the AD-related DMPs and the hippocampal burden of p-tau. On the other hand, and supporting the robustness of the present study, our results partially overlap those previously reported from methylome studies performed on other $\mathrm{AD}$ brain regions, such as prefrontal, frontal, and superior temporal neocortex or entorhinal cortex [9-14]. The overlap of DMPs among different AD brain regions suggests that a limited number of DMPs may be related to characteristic molecular processes of $\mathrm{AD}$, regardless of the affected brain area. In any case, our complete set of AD-related DMPs extends and complements the current epigenomic landscape of the AD brain.

Interestingly, the functional analysis showed that a significant percentage of the differentially methylated genes were related to neural development and neurogenesis. It was astounding that other biological, cellular, and molecular processes generally associated with neurodegeneration such as apoptosis, autophagy, inflammation, oxidative stress, and mitochondrial or lysosomal dysfunction were not overrepresented in the set of AD-related DMPs. Though strongly related to brain development, neurogenesis is also maintained in the adult human brain, mainly in two distinct areas, i.e., the subventricular zone and the subgranular zone of the dentate gyrus in the hippocampus. There is substantial neurogenesis throughout life in the human hippocampus as it is estimated that up to one third of human hippocampal neurons are subject to constant turnover [35]. Adult neurogenesis is linked to hippocampal-dependent learning and memory tasks [36-38] and is reduced during aging [35, 39]. Recent evidence suggests that adult neurogenesis is altered in the neurodegenerative process of $\mathrm{AD}$ [40-42], but it is still controversial with some authors reporting increased neurogenesis [43, 44], whereas others show reduced neurogenesis [39, 42, 45, 46]. In the human hippocampus, a sharp drop in adult neurogenesis has been observed in subjects with AD [42]. Remarkably, protein tau has been also involved in the modulation of adult hippocampal neurogenesis exerted by external stimuli [47] and impairs proliferation of neuronal precursors in the hippocampal dentate gyros in a tauopathy mouse model [48]. Definitely, the molecular mechanisms involved in defective neurogenesis in $\mathrm{AD}$ remain to be elucidated [41].

In this scenario, the results of the present study point to neurogenesis-related genes as targets of epigenetic changes in the hippocampus affected by AD. Enrichment of AD-related DNA methylation marks in neurodevelopmental and neurogenesis-related genes may reflect changes in epigenetic regulation of the neuronal population subjected to exchange in the hippocampus, whose function and balance could be relevant to $\mathrm{AD}$ pathogenesis. These methylation changes might be built throughout life due to external and internal cues and would represent an example of epigenetic interaction between environmental and genetic factors in developing $\mathrm{AD}$. As an alternative explanation, these epigenetic marks might also represent the trace of DNA methylation alterations induced during early developmental stages of the hippocampus, which would remain as a fingerprint in the larger proportion of hippocampal neurons that are not exchanged. This second hypothesis would link AD to early life stages, in concordance with recent studies that revealed abnormal p-tau deposits (pre-tangles) in brains of young individuals under $30[49,50]$ suggesting AD pathology would start earlier in life than it was previously thought. The influence of the genetic risk for $\mathrm{AD}$ has also been postulated to begin in early life [51], and other AD risk factors may be influenced by in utero environment [52].

We also observed that AD-related DMPs overlap relevant regulatory regions in the genome, such as $\mathrm{CpG}$ islands and bivalent histone marks corresponding to poised promoters. This result is in line with previous studies that found hypermethylated DMPs in the AD superior temporal gyrus to be enriched in poised promoters [14]. Promoters may be found in three distinct states: active, repressed, and poised. In the poised state, promoters are repressed but may be rapidly activated in response to certain cues [24]. Interestingly, poised promoters are very often associated with genes critical to the development and, as such, are characteristic of stem cells [24-27]. A number of poised promoters can also be maintained in lineage-committed and differentiated cells [29-31]. In our study, ChIP-qPCR analysis suggests that the bivalent promoters enriched in the set of AD-related DMPs resolve to a variety of states in committed NPCs and therefore are not enriched in genes retaining bivalent marks in lineage-committed cells.

The functional in silico analysis showed enrichment for the homeobox domain-containing family of genes in the set of AD-related DMPs. Homeobox domain-containing genes encode transcription factors (TFs) that result crucial during early embryonic development and morphogenesis [33]. Some homeobox TFs act by inducing cellular differentiation while other homeobox TFs are involved in maintaining pluripotency. Homeobox genes are known to be tightly regulated by DNA methylation and modifications of the chromatin state. Notably, homeobox domain-containing TFs are being closely connected to neurogenesis [53-55] and specific homeobox TFs, such as Dbx2, are involved in age-related neurogenic decline [56]. Most interestingly, Dbx2 is involved in the molecular changes that characterize the aged phenotype of neural stem/progenitor cells from the subventricular zone in mice, and 
it has been proposed as a player in promoting age-related neurogenic decline [56].

Deregulation of homeobox genes is related to certain diseases, such as cancer. However, the relationship with $\mathrm{AD}$ and neurodegenerative disorders has been barely assessed. Only a few reports are found in the literature regarding $\mathrm{AD}$ and homeobox domain-containing genes, e.g., low expression of $G A X$ gene, a regulator of vascular differentiation, in brain endothelial cells in AD [57], or GTX gene, a homeobox gene with neuroprotective properties [58]. Therefore, these findings open a new avenue for research to better understand the role of homeobox TFs in the AD pathogenesis.

At any rate, we want to be cautious with our conclusions. There was a significant difference in age between controls and $\mathrm{AD}$ patients, being the latter group older than the former group. Although we adjusted for age in the statistical differential methylation analysis, the accuracy of this correction may be limited as there is little overlap in the age ranges of both groups. For the sake of external validity, these findings should be replicated in an independent cohort of human hippocampal samples.

\section{Conclusions}

On the whole, our results suggest that altered DNA methylation in the AD hippocampus occurs at specific regulatory regions that are crucial for neural differentiation and support the notion that adult hippocampal neurogenesis may play a role in the development of AD. However, we are far from understanding how DNA methylation changes, interacting with other epigenetic mechanisms, modulate relevant molecular pathways in developing the disease. In addition, other topics such as the role of non-CpG methylation or hydroxymethylation would be interesting to address in the AD hippocampus. Therefore, further research on the alterations of epigenetic mechanisms in AD is guaranteed.

\section{Methods}

\section{Aim, design, and setting of the study}

The aim of this study was to profile genome-wide DNA methylation in the human hippocampus, a brain region particularly vulnerable to $\mathrm{AD}$ and the core of pathological protein tau deposits. This is an observational, transversal, case-control study to identify differentially methylated positions among AD cases and controls.

Human brain samples and neuropathological examination We evaluated postmortem hippocampal samples from 38 subjects (26 AD patients and 12 controls), provided by the Navarrabiomed Brain Bank. After death, half brain specimens from donors were cryopreserved at $-80^{\circ} \mathrm{C}$. Neuropathological examination was performed following the usual recommendations [59]. Assessment of $\beta$-amyloid deposit was carried out by immunohistochemical staining of paraffin-embedded sections (3-5 $\mu \mathrm{m}$ thick) with a mouse monoclonal (S6F/3D) anti- $\beta$-amyloid antibody (dilution 1/50) (Leica Biosystems Newcastle Ltd, Newcastle upon Tyne, UK). Evaluation of neurofibrillary pathology was performed with a mouse monoclonal antibody anti-human PHF-TAU, clone AT-8 (Tau AT8) (dilution 1/ 1000) (Innogenetics, Gent, Belgium), which identifies p-tau [19]. The reaction product was visualized using an automated slide immunostainer (Leica Bond Max) with Bond Polymer Refine Detection (Leica Biosystems Newcastle Ltd). AD staging was performed by using the ABC score according to the updated National Institute on Aging-Alzheimer's Association guidelines [60]. Agreement for any diagnosis was reached by members of a panel composed of two neuropathologists (VZ, CE) and two neurologists (JS, MM).

\section{Genome-wide DNA methylation profiling and differential methylation analysis}

CpG methylation levels were profiled genome-wide by using Infinium HumanMethylation450 BeadChip array (Illumina, Inc., San Diego, CA, USA) [61] at the Roswell Park Cancer Institute Genomics Shared Resource (Buffalo, NY, USA). Briefly, $500 \mathrm{ng}$ of genomic DNA from each brain sample was bisulfite treated and hybridized to the BeadChip according to the manufacturer's protocol. A total of 485,577 cytosine positions were interrogated throughout the human genome, covering the $99 \%$ of RefSeq genes and 96\% of CpG islands.

\section{Quality control and data processing}

In order to minimize the potential bias introduced by batch effects, we performed samples-to-batch allocation using the OSAT tool [62]. Microarray image processing was carried out using Genome Studio Methylation Module (v1.8.5). Background was corrected, and adjustment was performed to avoid type I/II assay chemistry bias. So as to minimize technical variation and improve data quality, the Dasen method [63] was used as a normalization tool.

Before performing differential methylation analysis, we removed probes that overlapped common single nucleotide polymorphisms (SNPs) and also those probes classified as internal controls of the Illumina microarray. Additionally, probes located on the $\mathrm{X}$ and $\mathrm{Y}$ chromosomes were discarded along with those probes previously described to hybridize to multiple locations in the genome $[64,65]$. Probes that technically did not pass the Illumina quality threshold (1188 probes with bead count $<3$ in $>5 \%$ of samples and 378 probes having $1 \%$ of samples with a detection $p$ value $>0.05$ ) were also removed. In the end, a total of 264,031 probes (representing individual CpG sites) were further analyzed for differential methylation (Additional file 1: Figure S3). 


\section{Differential methylation analysis}

Our aim was to identify differentially methylated positions (DMPs), that is to say, differentially methylated CpGs related with $\mathrm{AD}$ status. Linear model of microarray analysis (LIMMA) adjusted for age was performed to fit a linear regression model for each $\mathrm{CpG}$ site (R/Bioconductor package) [66]. Percentage of methylation ( $\beta$-value) at each surveyed $\mathrm{CpG}$ site was calculated and ranged from 0 to 1 . Benjamini and Hochberg false discovery rate (FDR) correction was used ( $p$ value $<0.05$ ). Methylation differences were prioritized by lowest adjusted $p$ values to ensure the most consistent DMPs between $\mathrm{AD}$ patients and controls. This analysis identified sets of candidate loci with consistent differences in methylation in AD versus control hippocampus. Gene annotation was obtained using the Genomic Regions Enrichment of Annotations Tool (GREAT) [31].

\section{Bisulfite sequencing validation of DMPs}

Next, $500 \mathrm{ng}$ of genomic DNA was bisulfite converted using the EpiTect Bisulfite Kit (QIAGEN, Redwood City, CA, USA) according to the manufacturer's instructions. Primer pair sequences were designed by MethPrimer [67] and are listed in Additional file 1: Table S6. PCR products were cloned using the TopoTA Cloning System (Invitrogen, Carlsbad, CA, USA), and between 12 and 24 independent clones were sequenced for each examined subject and region by Sanger sequencing [68]. Methylation graphs were obtained by using QUMA software [69], and maps of genes were drawn by using IGV software.

\section{Quantitative assessment of p-tau deposits in the hippocampus}

In order to quantitatively assess p-tau burden in the hippocampal samples of $\mathrm{AD}$ subjects, we applied a method described in detail elsewhere [7]. Briefly, sections of the hippocampus were examined after performing immunostaining with anti-p-tau antibody (clone AT-8) (dilution 1/ 1000), and representative images were analyzed with ImageJ software to obtain an average quantitative measure of the global p-tau deposit for each section and patient. Examples of AT-8 staining for control and different AD stages are shown in Additional file 1: Figure S4.

\section{Chromatin immunoprecipitation in Normal Human Neural Progenitor Cells}

Normal Human Neural Progenitor (NHNP) cells (Lonza) were grown in $75 \mathrm{~cm}^{2}$ culture flasks in NPBM medium (Neural Progenitor Basal Medium, Lonza) with the addition of hFGF, hEGF, NSF-1, and GA. NHNP cells were fixed in $1 \%$ formaldehyde for $10 \mathrm{~min}$ at room temperature; the reaction was stopped by addition of $1.25 \mathrm{M}$ glycine solution, and cells were washed in PBS and harvested in IP buffer (1 volume of SDS buffer to 0.5 volume of Triton dilution buffer and protease inhibitors).
For each ChIP, $100 \mu \mathrm{g}$ of DNA was used. Chromatin was sonicated to an average size of $750 \mathrm{bp}$. Sonicated sample was then blocked by incubating with Protein $\mathrm{G}$ and A sepharose beads at $4{ }^{\circ} \mathrm{C}$ for $1 \mathrm{~h}$. Ten percent of the sample was kept aside as INPUT, and $1 \mu \mathrm{g}$ of antibody or IgG was added to the remaining sample and incubated overnight at $4{ }^{\circ} \mathrm{C}$. The next day Protein $\mathrm{G}$ and $\mathrm{A}$ sepharose beads were added and incubated for $2 \mathrm{~h}$ at $4{ }^{\circ} \mathrm{C}$. After extensive washes, immunocomplexes were eluted from the beads and cross-links were reversed. The DNA was recovered by phenol-chloroform extraction and ethanol precipitation. DNA was resuspended in $150 \mu \mathrm{l}$ of water, and $3.75 \mu \mathrm{l}$ were used for real-time qPCRs in a final volume of $10 \mu \mathrm{l}$. The antibodies used in this study were anti-H3 (ab1791, Abcam), anti-H3K27me3 (ab6002, Abcam), and antiH3K4me3 (ab8580, Abcam). ChIP of the CCNL1 promoter and gene body regions were used as a positive control for active promoter histone marks (H3K4me3) and negative control for repressive histone marks (H3K27me3).

\section{Functional in silico analysis of DMPs}

We performed a systematic manual curation of the literature using PubMed to identify whether AD-related differentially methylated genes were enriched in nervous system functions, including neurogenesis and neural development.

In order to determine the biological significance of AD-related DMPs, gene ontology analysis and pathway analysis were performed using the Genomic Regions Enrichment of Annotations Tool (GREAT) [31]. To define gene regulatory domains, each gene was assigned a basal regulatory domain of a minimum distance upstream (5.0 $\mathrm{kb})$ and downstream of the transcription start site (TSS) $(1 \mathrm{~Kb}$ plus distal up to $1000 \mathrm{~Kb})$. The gene regulatory domain was extended in both directions to the nearest gene's basal domain but no more than the maximum extension in one direction. By using GREAT, we got InterPro ontology which contains data on protein domains, families, and functional sites. InterPro annotations give information about the function, structure, and evolution of the domains by combining several other databases (PROSITE, PRINTS, Pfam, ProDom, SMART, TIGRFAMs, PIRSF, SUPERFAMILY, PANTHER, and Gene3D). Only those terms with a FDR-corrected $p$ value less than 0.05 were reported.

\section{Additional file}

Additional file 1: Table S1. Brain sample set analyzed by $450 \mathrm{~K}$ Illumina BeadChip array. Table S2. Differentially methylated genes in previous AD methylome studies. Table S3. Correlation between 450K array data and DNA methylation levels obtained by bisulfite cloning sequencing. Table S4. Correlation between DNA methylation levels at each DMPs and tau burden. Table S5. InterPro Gene Ontology enrichment analysis. Table S6. Bisulfite PCR primers. Figure S1. Validation and extended mapping for the differentially methylated genes RHOB and NXN. Figure S2. Functional in silico study of 
DMPs. Figure S3. Bioinformatics pipeline. Figure S4. Representative examples of tau staining (AT8) for control and AD stages. (PDF $886 \mathrm{~kb}$ )

\section{Abbreviations}

AD: Alzheimer's disease; bp: Base pair; ChIP: Chromatin immunoprecipitation; CpG: Cytosine-phosphate-guanine dinucleotide; DMPs: Differentially methylated positions; GO: Gene ontology; GREAT: Genomic Regions Enrichment of Annotations Tool; GWAS: Genome-wide association studies; H1hESC: H1 human Embryonic Stem Cells; NH-A: Normal human astrocytes; NHNP: Normal Human Neural Progenitor Cells; NPBM: Neural Progenitor Basal Medium; OSAT: Optimal Sample Assignment Tool; PMI: Postmortem interval; p-tau: Hyperphosphorylated tau; SD: Standard deviation; SNPs: Single nucleotide polymorphisms; STRING: Search Tool for the Retrieval of Interacting Genes; TF: Transcription factor; TSS: Transcription start site; UCSC: University of California, Santa Cruz

\section{Acknowledgements}

We want to kindly thank Isabel Gil M.D., Ph.D., Ana Purroy, Valle Coca, and Ana Aramendía from the Navarrabiomed BrainBank for their technical support and help and Genomics Shared Resource of the Roswell Park Cancer Institute (Buffalo, NY, USA). We are very thankful to Teresa Tuñón M.D., Ph.D., and Federico García Bragado M.D., Ph.D., for their encouragement and anatomopathological support. We also want to thank Pitu Manubens + M.D., Ph.D., for invaluable mentoring and inspiration. Finally, we are very grateful to the patients and relatives that generously donor the brain tissue to the Navarrabiomed Brain Bank.

\section{Funding}

This work was supported by the Spanish Government through grants from the Institute of Health Carlos III (FIS PI13/02730 and PI17/02218), jointly funded by European Regional Development Fund (ERDF), European Union, "A way of shaping Europe"; the Regional Basque Government through a grant from The Basque Foundation for Health Innovation and Research (BIOEF) (BIO12/ALZ/007); a grant from Fundación Caja-Navarra and the Trans-Pyrenean Biomedical Research Network (REFBIO); crowdfunding through the PRECIPITA platform (Spanish foundation for science and technology-FECYT); and funding from "LaCaixa Foundation". In addition, AUC received a grant "Doctorados industriales 20182020" founded by Government of Navarra and MM received a grant "Programa de intensificación" founded by Fundación Bancaria "la Caixa" and Fundación CajaNavarra.

\section{Availability of data and materials}

All relevant data generated or analyzed during this study are included in this published article and its supplementary information files.

\section{Authors' contributions}

MA contributed to study design, acquisition of data, subject recruitment, data analysis and characterization, and drafting/revising the manuscript for content. AUC contributed to developing epigenetic experiments, analysis, and drawing figures. JSR contributed to drafting/revising the manuscript for content, analysis and interpretation of data (p-tau), and acquisition of image data. MVZ participated in acquiring data, revising subject diagnosis, and classifying patients and contributed to drafting/revising the manuscript for content. AL contributed to bioinformatics analysis and drafting the manuscript. JMJL performed ChIP experiments and statistical analysis. MR contributed to drawing figures, performed bisulfite experiments, and was involved in interpretation of data. IBL contributed to data analysis and to draft the manuscript for content. AP contributed to bioinformatics analysis. RL contributed to subject recruitment and characterization. IJ was involved in interpretation of data and contributed to drafting/revising the manuscript for content. CE participated in acquiring data, revising subject diagnosis, and classifying patients and contributed to the drafting/revising the manuscript for content. IML contributed to the study concept and design, obtaining funding, analysis of data and drafting/revising the manuscript for content. LDS contributed to ChIP experiments and drafting/revising the manuscript for content. MM contributed to study concept and design, subject recruitment and characterization, obtaining funding, study supervision, data analysis, and drafting/revising the manuscript for content. All authors read and approved the final manuscript.

\section{Ethics approval and consent to participate}

This study was carried out in accordance with the principles of the Declaration of Helsinki, and handling of human brain samples was performed according to the current Spanish national legislation (Royal Decree RD1716/2011). Written informed consent was obtained from all subjects or next of kin. The study was approved by the Ethics Committee of the Complejo Hospitalario de Navarra (90/2014) and the Scientific Committee of Navarrabiomed Brain Bank, Spain.

\section{Consent for publication}

Not applicable.

\section{Competing interests}

The authors declare that they have no competing interests.

\section{Publisher's Note}

Springer Nature remains neutral with regard to jurisdictional claims in published maps and institutional affiliations.

\section{Author details}

${ }^{1}$ Neuroepigenetics Laboratory, Navarrabiomed, Public University of Navarre (UPNA), IdiSNA (Navarra Institute for Health Research), c/ Irunlarrea, 3, 31008 Pamplona, Spain. ${ }^{2}$ Department of Neurology, Complejo Hospitalario de Navarra, IdiSNA (Navarra Institute for Health Research), Pamplona, Spain. ${ }^{3}$ Department of Pathology, Complejo Hospitalario de Navarra- IdiSNA (Navarra Institute for Health Research), Pamplona, Spain. ${ }^{4}$ Bioinformatics Unit, Navarrabiomed, Public University of Navarre (UPNA), IdiSNA (Navarra Institute for Health Research), Pamplona, Spain. ${ }^{5}$ Laboratoire de biologie cellulaire et moléculaire du contrôle de la prolifération (LBCMCP), Université Paul Sabatier, CNRS, Toulouse, France. ${ }^{6}$ Department of Internal Medicine, Hospital García-Orcoyen, Estella, Spain.

Received: 14 February 2019 Accepted: 25 April 2019

Published online: 19 June 2019

\section{References}

1. Winblad B, Amouyel P, Andrieu S, Ballard C, Brayne C, Brodaty H, et al. Defeating Alzheimer's disease and other dementias: a priority for European science and society. Lancet Neurol. 2016;15(5):455-532. https://doi.org/10. 1016/S1474-4422(16)00062-4.

2. Blennow K, de Leon MJ, Zetterberg H. Alzheimer's disease. Lancet. 2006; 368(9533):387-403. https://doi.org/10.1016/50140-6736(06)69113-7.

3. Reitz C, Brayne C, Mayeux R. Epidemiology of Alzheimer disease. Nat Rev Neurol. 2011;7(3):137-52. https://doi.org/10.1038/nrneurol.2011.2.

4. Sanchez-Mut JV, Graff J. Epigenetic alterations in Alzheimer's disease. Front Behav Neurosci. 2015;9:347. https://doi.org/10.3389/fnbeh.2015.00347.

5. Rao JS, Keleshian VL, Klein S, Rapoport SI. Epigenetic modifications in frontal cortex from Alzheimer's disease and bipolar disorder patients. Transl Psychiatry. 2012;2:e132. https://doi.org/10.1038/tp.2012.55.

6. Siegmund KD, Connor CM, Campan M, Long TI, Weisenberger DJ, Biniszkiewicz D, et al. DNA methylation in the human cerebral cortex is dynamically regulated throughout the life span and involves differentiated neurons. PLoS One. 2007;2(9):e895. https://doi.org/10.1371/journal.pone. 0000895.

7. Celarain N, Sanchez-Ruiz de Gordoa J, Zelaya MV, Roldan M, Larumbe R, Pulido $L$, et al. TREM2 upregulation correlates with 5-hydroxymethycytosine enrichment in Alzheimer's disease hippocampus. Clin Epigenetics. 2016;8:37. https://doi.org/10.1186/s13148-016-0202-9.

8. Mendioroz M, Celarain N, Altuna M, Sanchez-Ruiz de Gordoa J, Zelaya MV, Roldan M, et al. CRTC1 gene is differentially methylated in the human hippocampus in Alzheimer's disease. Alzheimers Res Ther. 2016;8(1):15. https://doi.org/10.1186/s13195-016-0183-0.

9. Bakulski KM, Dolinoy DC, Sartor MA, Paulson HL, Konen JR, Lieberman AP, et al. Genome-wide DNA methylation differences between late-onset Alzheimer's disease and cognitively normal controls in human frontal cortex. J Alzheimers Dis. 2012;29(3):571-88. https://doi.org/10.3233/jad-2012111223.

10. Sanchez-Mut JV, Aso E, Heyn H, Matsuda T, Bock C, Ferrer I, et al. Promoter hypermethylation of the phosphatase DUSP22 mediates PKA-dependent TAU phosphorylation and CREB activation in Alzheimer's disease. Hippocampus. 2014;24(4):363-8. https://doi.org/10.1002/hipo.22245. 
11. Lunnon K, Smith R, Hannon E, De Jager PL, Srivastava G, Volta M, et al. Methylomic profiling implicates cortical deregulation of ANK1 in Alzheimer's disease. Nat Neurosci. 2014;17(9):1164-70. https://doi.org/10.1038/nn.3782.

12. De Jager PL, Srivastava G, Lunnon K, Burgess J, Schalkwyk LC, Yu L, et al. Alzheimer's disease: early alterations in brain DNA methylation at ANK1, BIN1, RHBDF2 and other loci. Nat Neurosci. 2014;17(9):1156-63. https://doi. org/10.1038/nn.3786

13. Yu L, Chibnik LB, Srivastava GP, Pochet N, Yang J, Xu J, et al. Association of Brain DNA methylation in SORL1, ABCA7, HLA-DRB5, SLC24A4, and BIN1 with pathological diagnosis of Alzheimer disease. JAMA Neurol. 2015;72(1): 15-24. https://doi.org/10.1001/jamaneurol.2014.3049.

14. Watson CT, Roussos P, Garg P, Ho DJ, Azam N, Katsel PL, et al. Genomewide DNA methylation profiling in the superior temporal gyrus reveals epigenetic signatures associated with Alzheimer's disease. Genome Med. 2016;8(1):5. https://doi.org/10.1186/s13073-015-0258-8.

15. Klein HU, Bennett DA, De Jager PL. The epigenome in Alzheimer's disease: current state and approaches for a new path to gene discovery and understanding disease mechanism. Acta Neuropathol. 2016. https://doi.org/ 10.1007/s00401-016-1612-7.

16. Delgado-Morales R, Esteller M. Opening up the DNA methylome of dementia. Mol Psychiatry. 2017. https://doi.org/10.1038/mp.2016.242.

17. Wenk GL. Neuropathologic changes in Alzheimer's disease. J Clin Psychiatry. 2003;64(Suppl 9):7-10

18. Braak H, Braak E. Neuropathological stageing of Alzheimer-related changes. Acta Neuropathol. 1991;82(4):239-59.

19. Braak H, Alafuzoff I, Arzberger T, Kretzschmar H, Del Tredici K. Staging of Alzheimer disease-associated neurofibrillary pathology using paraffin sections and immunocytochemistry. Acta Neuropathol. 2006;112(4):389-404. https://doi.org/10.1007/s00401-006-0127-z.

20. Lace G, Savva GM, Forster G, de Silva R, Brayne C, Matthews FE, et al. Hippocampal tau pathology is related to neuroanatomical connections: an ageing population-based study. Brain. 2009;132(Pt 5):1324-34. https://doi. org/10.1093/brain/awp059

21. Guintivano J, Aryee MJ, Kaminsky ZA. A cell epigenotype specific model for the correction of brain cellular heterogeneity bias and its application to age, brain region and major depression. Epigenetics. 2013;8(3):290-302. https:// doi.org/10.4161/epi.23924.

22. Zhou X, Maricque B, Xie M, Li D, Sundaram V, Martin EA, et al. The human epigenome browser at Washington University. Nat Methods. 2011;8(12): 989-90. https://doi.org/10.1038/nmeth.1772.

23. Kent WJ, Sugnet CW, Furey TS, Roskin KM, Pringle TH, Zahler AM, et al. The human genome browser at UCSC. Genome Res. 2002;12(6):996-1006. https:/ doi.org/10.1101/gr.229102 Article published online before print in May 2002.

24. Harikumar A, Meshorer E. Chromatin remodeling and bivalent histone modifications in embryonic stem cells. EMBO Rep. 2015;16(12):1609-19. https://doi.org/10.15252/embr.201541011.

25. Bernstein BE, Mikkelsen TS, Xie X, Kamal M, Huebert DJ, Cuff J, et al. A bivalent chromatin structure marks key developmental genes in embryonic stem cells. Cell. 2006;125(2):315-26. https://doi.org/10.1016/j.cell.2006.02.041.

26. Zhao XD, Han X, Chew JL, Liu J, Chiu KP, Choo A, et al. Whole-genome mapping of histone $\mathrm{H} 3$ Lys4 and 27 trimethylations reveals distinct genomic compartments in human embryonic stem cells. Cell Stem Cell. 2007;1(3):286-98. https://doi.org/10.1016/.stem.2007.08.004

27. Abraham BJ, Cui K, Tang Q, Zhao K. Dynamic regulation of epigenomic landscapes during hematopoiesis. BMC Genomics. 2013;14:193. https://doi. org/10.1186/1471-2164-14-193.

28. Mikkelsen TS, Ku M, Jaffe DB, Issac B, Lieberman E, Giannoukos G, et al. Genome-wide maps of chromatin state in pluripotent and lineage-committed cells. Nature. 2007:448(7153):553-60. https://doi.org/10.1038/nature06008.

29. Maupetit-Méhouas S, Montibus B, Nury D, Tayama C, Wassef M, Kota SK, et al. Imprinting control regions (ICRs) are marked by mono-allelic bivalent chromatin when transcriptionally inactive. Nucleic Acids Res. 2016;44(2):62135. https://doi.org/10.1093/nar/gkv960.

30. Yoo S, Bieda MC. Differences among brain tumor stem cell types and fetal neural stem cells in focal regions of histone modifications and DNA methylation, broad regions of modifications, and bivalent promoters. BMC Genomics. 2014;15:724. https://doi.org/10.1186/14712164-15-724.

31. McLean CY, Bristor D, Hiller M, Clarke SL, Schaar BT, Lowe CB, et al. GREAT improves functional interpretation of cis-regulatory regions. Nat Biotechnol. 2010;28(5):495-501. https://doi.org/10.1038/nbt.1630.
32. Thomas JO. HMG1 and 2: architectural DNA-binding proteins. Biochem Soc Trans. 2001;29(Pt 4):395-401.

33. Corsetti MT, Briata P, Sanseverino L, Daga A, Airoldi I, Simeone A, et al. Differential DNA binding properties of three human homeodomain proteins. Nucleic Acids Res. 1992;20(17):4465-72.

34. Serrano-Pozo A, Frosch MP, Masliah E, Hyman BT. Neuropathological alterations in Alzheimer disease. Cold Spring Harb Perspect Med. 2011;1(1): a006189. https://doi.org/10.1101/cshperspect.a006189.

35. Spalding KL, Bergmann O, Alkass K, Bernard S, Salehpour M, Huttner HB, et al. Dynamics of hippocampal neurogenesis in adult humans. Cell. 2013; 153(6):1219-27. https://doi.org/10.1016/j.cell.2013.05.002.

36. Dokter $\mathrm{M}$, von Bohlen und Halbach O. Neurogenesis within the adult hippocampus under physiological conditions and in depression. Neural Regen Res. 2012;7(7):552-9. https://doi.org/10.3969/j.issn.1673-5374.2012.07.013.

37. Ming GL, Song H. Adult neurogenesis in the mammalian brain: significant answers and significant questions. Neuron. 2011;70(4):687-702. https://doi. org/10.1016/j.neuron.2011.05.001.

38. Drapeau E, Mayo W, Aurousseau C, Le Moal M, Piazza PV, Abrous DN. Spatial memory performances of aged rats in the water maze predict levels of hippocampal neurogenesis. Proc Natl Acad Sci U S A. 2003;100(24):1438590. https://doi.org/10.1073/pnas.2334169100.

39. Mathews K, Allen KM, Boerrigter D, Ball H, Shannon Weickert C, Double KL. Evidence for reduced neurogenesis in the aging human hippocampus despite stable stem cell markers. Aging Cell. 2017. https://doi.org/10.1111/ acel.12641.

40. Mu Y, Gage FH. Adult hippocampal neurogenesis and its role in Alzheimer's disease. Mol Neurodegener. 2011;6:85. https://doi.org/10.1186/1750-1326-6-85.

41. Crews L, Masliah E. Molecular mechanisms of neurodegeneration in Alzheimer's disease. Hum Mol Genet. 2010;19(R1):R12-20. https://doi.org/10. 1093/hmg/ddq160.

42. Moreno-Jimenez EP, Flor-Garcia M, Terreros-Roncal J, Rabano A, Cafini F, PallasBazarra N, et al. Adult hippocampal neurogenesis is abundant in neurologically healthy subjects and drops sharply in patients with Alzheimer's disease. Nat Med. 2019. https://doi.org/10.1038/s41591-019-0375-9.

43. Jin K, Peel AL, Mao XO, Xie L, Cottrell BA, Henshall DC, et al. Increased hippocampal neurogenesis in Alzheimer's disease. Proc Natl Acad Sci U S A 2004;101(1):343-7. https://doi.org/10.1073/pnas.2634794100.

44. Gomez-Nicola D, Suzzi S, Vargas-Caballero M, Fransen NL, Al-Malki H, Cebrian-Silla A, et al. Temporal dynamics of hippocampal neurogenesis in chronic neurodegeneration. Brain. 2014;137(Pt 8):2312-28. https://doi.org/10 1093/brain/awu155

45. Boekhoorn K, Joels M, Lucassen PJ. Increased proliferation reflects glial and vascular-associated changes, but not neurogenesis in the presenile Alzheimer hippocampus. Neurobiol Dis. 2006;24(1):1-14. https://doi.org/10. 1016/j.nbd.2006.04.017.

46. Li B, Yamamori H, Tatebayashi Y, Shafit-Zagardo B, Tanimukai H, Chen S, et al. Failure of neuronal maturation in Alzheimer disease dentate gyrus. J Neuropathol Exp Neurol. 2008;67(1):78-84. https://doi.org/10.1097/nen. Ob013e318160c5db.

47. Pallas-Bazarra N, Jurado-Arjona J, Navarrete M, Esteban JA, Hernández F, Ávila J, et al. Novel function of Tau in regulating the effects of external stimuli on adult hippocampal neurogenesis. EMBO J. 2016;35(13):1417-36. https://doi.org/10.15252/embj.201593518.

48. Houben S, Leroy K, Ando K, Yilmaz Z, Widomski C, Buee L, et al. Genetic ablation of tau in postnatal neurons rescues decreased adult hippocampal neurogenesis in a tauopathy model. Neurobiol Dis. 2019;127:131-41. https:// doi.org/10.1016/j.nbd.2019.02.021.

49. Braak H, Del Tredici K. The pathological process underlying Alzheimer's disease in individuals under thirty. Acta Neuropathol. 2011;121(2):171-81. https://doi.org/10.1007/s00401-010-0789-4.

50. Braak H, Thal DR, Ghebremedhin E, Del Tredici K. Stages of the pathologic process in Alzheimer disease: age categories from 1 to 100 years. J Neuropathol Exp Neurol. 2011;70(11):960-9. https://doi.org/10.1097/NEN ob013e318232a379.

51. Mormino EC, Sperling RA, Holmes AJ, Buckner RL, De Jager PL, Smoller JW, et al. Polygenic risk of Alzheimer disease is associated with early- and latelife processes. Neurology. 2016;87(5):481-8. https://doi.org/10.1212/WNL. 0000000000002922

52. Ross MG, Desai M, Khorram O, McKnight RA, Lane RH, Torday J. Gestational programming of offspring obesity: a potential contributor to Alzheimer's disease. Curr Alzheimer Res. 2007;4(2):213-7. 
53. Estacio-Gomez A, Diaz-Benjumea FJ. Roles of Hox genes in the patterning of the central nervous system of Drosophila. Fly. 2014;8(1):26-32. https://doi. org/10.4161/fly.27424.

54. Karalay O, Doberauer K, Vadodaria KC, Knobloch M, Berti L, Miquelajauregui $\mathrm{A}$, et al. Prospero-related homeobox 1 gene (Prox1) is regulated by canonical Wnt signaling and has a stage-specific role in adult hippocampal neurogenesis. Proc Nat Acad Sci U S A. 2011;108(14):5807-12. https://doi. org/10.1073/pnas.1013456108.

55. Technau GM, Rogulja-Ortmann A, Berger C, Birkholz O, Rickert C. Composition of a neuromere and its segmental diversification under the control of Hox genes in the embryonic CNS of Drosophila. J Neurogenet. 2014;28(3-4):171-80. https://doi.org/10.3109/01677063.2013. 868459.

56. Lupo G, Nisi PS, Esteve P, Paul YL, Novo CL, Sidders B, et al. Molecular profiling of aged neural progenitors identifies $\mathrm{Db} \times 2$ as a candidate regulator of age-associated neurogenic decline. Aging Cell. 2018;17(3):e12745. https:// doi.org/10.1111/acel.12745.

57. Wu Z, Guo H, Chow N, Sallstrom J, Bell RD, Deane R, et al. Role of the MEOX2 homeobox gene in neurovascular dysfunction in Alzheimer disease. Nat Med. 2005;11(9):959-65. https://doi.org/10.1038/nm1287.

58. Hashimoto Y, Tsuji O, Kanekura K, Aiso S, Niikura T, Matsuoka M, et al. The Gtx homeodomain transcription factor exerts neuroprotection using its homeodomain. J Biol Chem. 2004;279(16):16767-77. https://doi.org/10.1074/ jbc.M313630200.

59. Bell JE, Alafuzoff I, Al-Sarraj S, Arzberger T, Bogdanovic N, Budka H, et al. Management of a twenty-first century brain bank: experience in the BrainNet Europe consortium. Acta Neuropathol. 2008;115(5):497-507. https://doi.org/10.1007/s00401-008-0360-8,

60. Montine TJ, Phelps CH, Beach TG, Bigio EH, Cairns NJ, Dickson DW, et al. National Institute on Aging-Alzheimer's Association guidelines for the neuropathologic assessment of Alzheimer's disease: a practical approach. Acta Neuropathol. 2012;123(1):1-11. https://doi.org/10.1007/ s00401-011-0910-3.

61. Sandoval J, Heyn H, Moran S, Serra-Musach J, Pujana MA, Bibikova M, et al. Validation of a DNA methylation microarray for $450,000 \mathrm{CpG}$ sites in the human genome. Epigenetics. 2011;6(6):692-702.

62. Yan L, Ma C, Wang D, Hu Q, Qin M, Conroy JM, et al. OSAT: a tool for sample-to-batch allocations in genomics experiments. BMC Genomics. 2012; 13:689. https://doi.org/10.1186/1471-2164-13-689.

63. Pidsley R, Y Wong CC, Volta M, Lunnon K, Mill J, Schalkwyk LC. A datadriven approach to preprocessing Illumina $450 \mathrm{~K}$ methylation array data. BMC Genomics. 2013;14:293.

64. Chen YA, Lemire M, Choufani S, Butcher DT, Grafodatskaya D, Zanke BW, et al. Discovery of cross-reactive probes and polymorphic CpGs in the lllumina Infinium HumanMethylation450 microarray. Epigenetics. 2013;8(2):203-9. https://doi.org/10.4161/epi.23470.

65. Price ME, Cotton AM, Lam LL, Farre P, Emberly E, Brown CJ, et al. Additional annotation enhances potential for biologically-relevant analysis of the Illumina Infinium HumanMethylation450 BeadChip array. Epigenetics Chromatin. 2013;6(1):4. https://doi.org/10.1186/17568935-6-4.

66. Ritchie ME, Phipson B, Wu D, Hu Y, Law CW, Shi W, et al. Limma powers differential expression analyses for RNA-sequencing and microarray studies. Nucleic Acids Res. 2015;43(7):e47. https://doi.org/10. 1093/nar/gkv007.

67. Li LC, Dahiya R. MethPrimer: designing primers for methylation PCRs. Bioinformatics. 2002;18(11):1427-31.

68. Sanger F, Nicklen S, Coulson AR. DNA sequencing with chain-terminating inhibitors. Proc Natl Acad Sci U S A. 1977:74(12):5463-7.

69. Kumaki Y, Oda M, Okano M. QUMA: quantification tool for methylation analysis. Nucleic Acids Res. 2008;36(Web Server issue):W170-5. doi:10.1093/ nar/gkn294.

Ready to submit your research? Choose BMC and benefit from:

- fast, convenient online submission

- thorough peer review by experienced researchers in your field

- rapid publication on acceptance

- support for research data, including large and complex data types

- gold Open Access which fosters wider collaboration and increased citations

- maximum visibility for your research: over $100 \mathrm{M}$ website views per year

At $\mathrm{BMC}$, research is always in progress.

Learn more biomedcentral.com/submissions 